\title{
Textos escolares: consideraciones didácticas
}

\author{
Raquel Soaje de Elías
}

orcid.org/0000-0003-0825-4454

Universidad de los Andes, Chile

rsoaje@uandes.cl

\section{Resumen}

La edición de los textos escolares en Chile pone en juego cada año grandes sumas de dinero, lo que la convierte en una industria muy apetecida. De alli la importancia crucial de indagar en qué medida los libros son coherentes con la finalidad didáctica en la cual se fundamentan. La investigación realizada supone una continuación del estudio acerca de la calidad de los textos estatales chilenos en el subsector de Historia y Ciencias Sociales, con el propósito de evaluar en qué medida en estos últimos años (2012-2016) se han producido cambios en la concepción y confección, que indiquen una mejora en su calidad y en su aporte como herramienta crucial de apoyo pedagógico. Entre los objetivos destaca el de señalar las principales fortalezas y debilidades de los textos seleccionados, tomando como variable su contenido, la cual será dimensionada en función de determinados indicadores de fondo y forma. Se seleccionó para ello una muestra del periodo 2012-2016 de textos editados o reeditados para el Ministerio de Educación (MINEDUC) en el año 2016; luego se definieron los indicadores para la dimensión de la variable y el análisis posterior; finalmente se expusieron los resultados, en función de los del estudio anterior. Como conclusión principal se constató la necesidad urgente de revisar ciertos aspectos esenciales: la equidad en el tratamiento de los contenidos, la objetividad en la presentación de hechos y fenómenos, así como una adecuada explicación de estos a partir de sus causas y efectos.

\section{Palabras clave}

Enseñanza de las ciencias sociales; calidad de la enseñanza; libros de texto; enseñanza de la historia; Chile (Fuente: Tesauro de la Unesco).

Recepción: 09/05/2017 | Envío a pares: 30/10/2017 | Aceptación por pares: 27/11/2017 | Aprobación: 30/11/2017 


\title{
School Textbooks: Didactic Considerations
}

\begin{abstract}
The publication of school textbooks in Chile puts large sums of money into play each year, which makes it a very desirable industry. This underscores the crucial importance of investigating to what extent these textbooks are consistent with the didactic purpose for which they are intended. This study is a continuation of previous research that was done on the quality of textbooks used by Chilean public schools in the history and social sciences subsector, so as to evaluate to what extent recent years (2012-2016) have seen changes in their conception and make-up that might indicate an improvement in their quality and contribution as a crucial tool to support the teaching process. One of the prime objectives was to identify the major strengths and weaknesses of the selected texts, using their content as a variable, which is dimensioned according to certain indicators of form and substance. A sample of textbooks from the period between 2012 and 2016 that were published or republished for the Ministry of Education (MINEDUC) in 2016 was selected for this purpose. Then, the indicators for the dimensions of the variable and the subsequent analysis were defined. The results were presented and keyed to those of the previous study. One of the main conclusions indicates there is an urgent need to review certain essential aspects; namely, impartiality in the way content is treated and objectivity in the presentation offacts and phenomena, as well as an adequate explanation based on their causes and effects.
\end{abstract}

\section{Keywords}

Social science education; Educational quality; Textbooks; History education (Source: Unesco Thesaurus). 


\section{Textos escolares: considerações didáticas}

\section{Resumo}

A edição dos livros didáticos no Chile movimenta todos os anos grandes quantidades de dinheiro, o que a converte numa indústria muito cobiçada. Portanto, é importante questionar em que medida os livros são coerentes com a finalidade didática na qual estão fundamentados. Esta pesquisa terminada supõe uma continuidade do estudo sobre a qualidade dos livros didáticos estatais chilenos no subsetor de História e Ciências Sociais, com o propósito de avaliar quais mudanças na sua concepção e elaboração foram produzidas entre 2012 e 2016, que indiquem uma melhoria em sua qualidade e em sua contribuição como ferramenta fundamental de apoio pedagógico. Entre os objetivos, destaca-se o de indicar as principais fortalezas e fraquezas dos livros selecionados, tomando como variável seu conteúdo, a qual será dimensionada em função de determinados indicadores de fundo e forma. Para isso, foi selecionada uma amostra do periodo 2012-2016 de livros editados ou reeditados para o Ministério da Educação em 2016. Em seguida, foram definidos os indicadores para a dimensão da variável e para a análise posterior; finalmente, foram expostos os resultados de acordo com o estudo anterior. Como conclusão principal, constatou-se a necessidade urgente de revisar aspectos essenciais como: equidade no tratamento dos conteúdos, objetividade na apresentação de fatos e fenômenos, bem como adequada explicação destes a partir de suas causas e efeitos.

\section{Palavras-chave}

Ensino das ciências sociais; qualidade da educação; livro didático; ensino da história; Chile (Fonte: Tesauro da Unesco). 


\section{Introducción}

El presente trabajo se inserta en el ámbito del estudio de los textos escolares, entendidos como herramientas fundamentales del quehacer didáctico en la actualidad. Su edición pone en juego cada año, al menos en Chile, grandes sumas de dinero por parte del Estado, pero también afecta el bolsillo de los particulares, tanto de instituciones educativas como de apoderados, por lo cual constituye una industria muy apetecida por las grandes editoriales locales y extranjeras. Cabe acotar en este sentido que el monto señalado en el presupuesto estatal para los textos escolares por la Dirección de Presupuestos del Ministerio de Hacienda (DIPRES, 2016) se fijó para el año 2016 en \$35.014.724, solamente para enseñanza básica y media, casi $75 \%$ más que el monto utilizado en 2010 (Soaje y Orellana, 2013). De allí la importancia crucial de analizar su contenido, para determinar en qué medida responden a la finalidad didáctica que ellos representan y en qué proporción se justifica el gasto de ambos sectores, público y privado, en esta materia.

Cabe acotar, por otra parte, que son escasos los estudios realizados acerca de los textos escolares que distribuye el Ministerio de Educación (Mineduc), siendo pionero el análisis realizado por investigadores del Centro de Estudios Públicos (Eyzaquirre y Fontaine, 1997), además de aquellos que encarga el Ministerio a diferentes universidades chilenas. Entre estos últimos, destaca el trabajo efectuado por el Centro de Microdatos (2013) de la Universidad de Chile acerca del uso de los textos por docentes y alumnos. El mismo arrojó ciertas conclusiones notables, como por ejemplo que el porcentaje de uso en clases del texto escolar en general se encuentra sobre el 90\%, pero mientras que en educación parvularia y básica su frecuencia de uso se mantiene en este porcentaje, en los ciclos superiores decae a un $76 \%$, siendo alto de todas maneras. Además, según una tabla de valoración de los textos escolares en una escala de 1 a 4 -donde 1 equivale a los textos menos valorados y 4 al más valorado- los textos de educación parvularia y de primer ciclo se encuen- tran sobre los 3 puntos en promedio, mientras que, a medida que se avanza en niveles hacia enseñanza media, el promedio de valoración comienza a decaer (Centro de Microdatos, 2013).

Estos datos se insertan a su vez en un panorama complejo para Chile, pues, aunque los resultados del Tercer Estudio Regional Comparativo y Explicativo (TERCE), realizado por la Unesco, así como de otras pruebas internacionales', señalan que Chile es el país con mejor ranking educacional de la región, a pesar de sus índices de pobreza y la poca inversión en educación, nuestra región es una de las que poseen peores resultados entre las evaluadas en este ámbito (Claro, 2015).

Respecto de nuestro estudio, la investigación supone una continuación de la indagación efectuada anteriormente acerca de la calidad de los textos en el área de Historia y Ciencias Sociales (Soaje, 2012), en la cual se establecieron algunas conclusiones que creemos necesario recordar, con el propósito de evaluar en qué medida en estos últimos cinco años (2011-2016) se han producido cambios en la concepción y confección de dichos textos que indiquen una mejora de su calidad y en su aporte como herramienta crucial de apoyo pedagógico. Los objetivos específicos son:

- Señalar las principales fortalezas y debilidades de los textos seleccionados, tomando como variable su contenido, la cual será dimensionada en función de ciertos indicadores que hacen referencia a su fondo y forma.

- Analizar la concepción de la Historia y su relación con las Ciencias Sociales que subyace a las propuestas editoriales, y cotejar con la que ofrecen las Bases Curriculares del 2013.

1 Programa Internacional para la Evaluación de Estudiantes (Programme for International Student Assessment - PISA) y Estudio de las Tendencias en Matemáticas y Ciencias (Trends in International Mathematics and Science Study - TIMSS). 
- Registrar las tendencias derivadas del comportamiento de la variable mencionada.

- Cotejar el resultado del análisis efectuado con las conclusiones del estudio anterior, a los fines de identificar elementos de continuidad y de cambio, así como los nuevos aportes que se han incorporado y las carencias que aún se pueden observar.

\section{Metodología}

Cabe señalar que este estudio utiliza una metodología principalmente cualitativa, sobre todo en el análisis de los datos obtenidos, basada en un tipo de estudio descriptivo y exploratorio, que tiene como fin evaluar la calidad de los textos escolares. Para ello he seleccionado una muestra de siete textos correspondientes al subsector Historia, Geografía y Ciencias Sociales, los cuales se ubican entre los grados $6^{\circ}$ Básico y $4^{\circ}$ Medio, y corresponden a ediciones del periodo 2012-2015², pero reeditadas en 2016. Se trata de una muestra discrecional o intencional, efectuada teniendo en cuenta los resultados de la última licitación disponible, en la cual se combinan cursos del segundo ciclo de enseñanza básica, con los correspondientes a los del nivel de educación media. Las casas editoriales que se adjudicaron dicha licitación estatal para cubrir la demanda propia del año 2016, de colegios municipales y particulares subvencionados, fueron: la española SM, para casi todos los textos, exceptuando el de $1^{\circ}$ Medio, otorgado a Santillana del Pacífico, también española, y el de $4^{\circ}$ Medio, adquirido por la editorial chilena ZigZag, única del ámbito local, al menos en los niveles seleccionados. El criterio de escogencia de la muestra se vincula al tipo de organización curricular que posee el último ciclo de la enseñanza básica, el cual funciona distinto al primer ciclo, más afín a la enseñanza media, con cursos separados por especialidad

2 Cabe acotar que los textos adjudicados para 2016 corresponden a ediciones del periodo mencionado. Es decir, se trata de reediciones efectuadas para cubrir la demanda de dicho año. y dictados generalmente por docentes con una formación académica de licenciados y/o profesores en la especialidad.

La primera etapa de este estudio comprendió la revisión de los textos mencionados, entregados por Mineduc a los alumnos entre los años 2012-2016, tomando como eje la variable de su contenido. En segundo término, la misma fue dimensionada a partir de diversos indicadores, especificados más adelante, los cuales permitieron establecer ciertas tendencias, indispensables para realizar una comparación con el estudio anterior (Soaje, 2012).

Para el análisis de los textos se aplicó una tabla con los indicadores correspondientes y de esta matriz se extrajeron tendencias observadas con base en los resultados obtenidos. En la exposición de los resultados se complementó el análisis con ejemplos específicos, extraídos de los textos estudiados, además de la necesaria triangulación con la bibliografía especializada. Se realizó luego el cotejo de los resultados con las conclusiones principales del estudio de Soaje (2012) que hacían referencia a la falta de profundidad en el tratamiento de los contenidos; carencia de equidad respecto a los temas tratados y sesgo ideológico existente en los manuales, lo cual redunda en perjuicio de la objetividad a la que aspiran las Bases Curriculares.

Cabe consignar, por último, que en el análisis de los textos actuales se tuvo en cuenta la política sobre textos escolares establecida por el Mineduc ${ }^{3}$, elaborada precisamente para orientar a los equipos editoriales que postulan a las licitaciones anuales efectuadas por dicho organismo, además de otros documentos ministeriales, como las Bases Curriculares de 2013 y las recientemente elaboradas para 2017. Todos estos documentos han permitido visualizar la perspectiva pedagógico-historiográfica con base en la cual se han estructurado los textos,

3 Sobre la política de textos escolares, véase Seminario Internacional Textos Escolares de Historia y Ciencias Sociales, Santiago de Chile. En línea. 
la cual analizaremos a continuación, pues la misma condiciona fuertemente su línea editorial, a la vez que constituye un factor importante para comprender dicha estructuración.

\section{La nueva enseñanza de la Historia: “¿Forjar patriotas o educar cosmopolitas?”}

Las Bases Curriculares y las introducciones de los textos escolares son fuentes inestimables para conocer la perspectiva de la nueva enseñanza de la Historia, que dichos documentos reflejan.

¿Qué es la Historia, Geografía y Ciencias sociales? Es el estudio y reflexión sobre la forma en que los seres humanos nos relacionamos a través del tiempo, la forma en que vivimos en sociedad actualmente en Chile y la forma en que nos vinculamos con el espacio y el ambiente en que habitamos, tanto en el pasado como en el presente. (Landa y Pinto, 2015b, p. 3)

Con estas palabras comienza el texto de $8^{\circ}$ Básico y de manera similar el de $7^{\circ}$ Básico, de la editorial SM, la cual ganó la licitación para la cobertura de dichos textos del año 2016. La pregunta, mal redactada -pues si son tres disciplinas debería utilizarse el verbo en plural y responder de la misma forma- sugiere que las tres disciplinas se consideran parte de un conjunto que las engloba, cuya definición intenta responder precisamente a esa pluralidad, teniendo presente las dimensiones espacio-temporales y, a la vez, el fenómeno social que se inserta en la conjunción de ambas. Ello nos induce a rememorar definiciones de la Historia como disciplina independiente, valiosa por su propio peso, que apuntan a otros aspectos propios de la reflexión acerca del pasado humano. Uno de ellos es la importancia de la memoria, base y fundamento de la necesidad de registrar los hechos, por parte del ser humano, expresado de manera explícita, al menos desde Heródoto hasta la actualidad. Baste como ejemplo de la trascendencia del concepto la creación en Chile del llamado Museo de la Memoria, cuyo propósito fue el recuerdo de los hechos reprobables del pasado reciente, o bien el Museo del Holocausto y tantos otros, locales e internacionales, testigos vivos de hechos considerados nefastos por sus creadores, que apuntan a educar a las generaciones nuevas según la experiencia acumulada por sus antepasados, durante ese pasado inmediato. La memoria es entonces la meta clave del aprendizaje de la Historia. Los antiguos lo tenían muy presente, e incluso la frase que Cicerón dejó impresa en una de sus obras, que hacía referencia a la historia como "testigo de los tiempos, luz de la verdad, vida de la memoria, maestra de la vida, heraldo del pasado"4, respondía a una idea común de su época. La transmisión de esta idea de generación en generación, tan cara a la concepción clásica de la enseñanza de la Historia, queda completamente relegada ante este nuevo enfoque didáctico.

Otro aspecto a destacar de aquella definición es que el ser individual y peculiar, que sobresale por sobre la masa, se diluye en la nueva concepción planteada, ya sea porque la relación social predomina por sobre la acción personal del sujeto implicado en su pasado o bien porque el modo de vida común es superior a las personas mismas que viven así. De manera similar, los procesos predominan sobre los hechos reales y los grupos humanos por sobre los seres concretos de carne y hueso. Solo el caso de científicos, artistas e intelectuales destacados permite mencionar individualidades, lo cual se justifica, en último término, por la función social que han cumplido. Lo humano entonces se diluye en categorías provenientes de la sociología o de la antropología, mientras la Historia se va desdibujando como disciplina independiente y su importancia se va esfumando dentro del conjunto de las nuevas "Ciencias Sociales".

Es una visión de la Historia, pero lo preocupante es que no se mencionan otras, que podrían darle al alumno un panorama más amplio sobre cómo

4 Al respecto, el texto latino dice "historia vero testis temporum, lux veritatis, vita memoriae, magistra vitae, nuntia vetustatis". 
abordar su estudio (González, 2005). La referencia al debate acerca de la Historia como ciencia o como disciplina humanística subyace a esta postura, consecuente con una visión histórica más descarnada, más analítica y cuantitativa, en la cual el lenguaje "científico" predomina por sobre otro más narrativo, que incluye personajes, hechos y fechas precisas (Aurell, Balmaceda, Burke y Soza, 2013). Se trata de la tensión derivada de dos posturas antagónicas, que han dado origen a dos perspectivas acerca de la enseñanza de esta disciplina desde los inicios de los Estados liberales y que, según observan Mario Carretero y Manuel Montanero, responden a "la racionalidad crítica de la llustración y la emotividad identitaria del Romanticismo" (2008, p. 135). Esta tensión se traduciría, en el mundo global en que vivimos, en los siguientes términos: “¡Forjar patriotas o educar cosmopolitas?" (Carretero y Kriger, 2004).

Un tercer elemento a considerar se fundamenta en la concepción del texto escolar como "herramienta para la generación de un conocimiento que se conciba y sea constructor de mundo y de historia" (Quintana, Castillo, Pérez, Moyano y Thielemann, 2012, p. 3). A la vez, se lo considera un aporte a la "democratización del conocimiento", concebida no en el sentido de una ampliación de la cantidad de información, en este caso, a disposición de los estudiantes, sino como una forma de aprehender la realidad, a partir de la reflexión y la toma de posiciones, crítica y fundamentada. Llama la atención en este enunciado del libro de $3^{\circ}$ Medio la falta de referencia a aquella finalidad que ha sido la base de la disciplina histórica desde Heródoto y Tucídides, considerado "padre de la historia" el primero y del "rigor histórico" el segundo, finalidad vinculada a la pretensión de narrar los hechos de manera veraz, tal como sucedieron en el pasado. Pero, además, también se plantea como propósito central desarrollar habilidades para evaluar críticamente los fenómenos históricos y, a la vez, ejercitar su capacidad para exponer los resultados de su investigación y sus opiniones de forma sintética y fundamentada (Quintana et al., 2012).
También se echa de menos la apetencia del rigor científico al que aspira todo aquel que se dedica a las ciencias llamadas sociales, ante la imposibilidad de establecer leyes universales a partir de sus investigaciones. Es este factor, sin embargo, el que otorgará la tan ansiada "objetividad", a la cual aspiran los inspiradores de estos manuales escolares.

Por último, la finalidad puramente utilitaria del texto, puesto al servicio de la "construcción de mundo y de historia", plantea un interrogante difícil de responder en una primera aproximación al estudio de dichos textos, y que se podría expresar en los siguientes términos: ¿Cuál es la concepción de Historia y de Ciencia que queda implícita en esta premisa? ¿Cuál es el sentido de la uniformidad, por la cual todos los alumnos deben compartir el mismo texto de estudio? ¿Por qué el alumno debería estudiar esta asignatura a partir de estos textos y no de otros diferentes? Si uno de los objetivos principales es estimular el pensamiento crítico, ¿por qué no fomentar el uso de diferentes textos a partir de los cuales habría más posibilidad de encontrar distintas visiones acerca del pasado y de la actualidad? ¿Cuáles son los criterios según los cuales se escoge una visión de la Historia en detrimento de otras?

Cabe acotar, sin embargo, que las Bases Curriculares (MIneduc, 2013) son coherentes con dichas premisas, puesto que su meta última apunta a entregar a los estudiantes aquellas herramientas "que les permitan ejercer como ciudadanos activos y respetuosos de los principios en los que se funda la democracia, y que desarrollen y practiquen una conciencia ética basada en los Derechos Humanos" (p. 194). Por otra parte, ponen el acento en la importancia de reconocer "la diversidad de visiones que pueden existir para aproximarse a la realidad social [...] [y que los alumnos] comprendan que la validez de las interpretaciones está sujeta a distintos criterios que buscan evitar el relativismo y promover una opinión rigurosa e informada" (Mineduc, 2013, p. 197). 
De este principio surge un nuevo interrogante acerca de dichos textos: ¿Los distintos temas tratados son presentados de acuerdo con las diferentes visiones que existen en la actualidad, con la misma intensidad, según se trate de una postura o de otra? Ejemplo de esto puede ser el periodo de la dictadura militar o bien el período previo y el subsiguiente, en los cuales "la diversidad de visiones" se reduce a escasas referencias a la posición de "los otros" (Fernández y Giadrosić, 2015, pp. 244-249). Sobre esto volveremos más adelante. Y en segundo lugar, ¿existe equidad en el tratamiento de los contenidos? ¿Por qué la Historia contemporánea posee una preeminencia absoluta, mientras las demás etapas quedan relegadas a un segundo plano? O bien ¿cuáles son los criterios que subyacen al énfasis puesto en ciertos temas, como los derechos femeninos, los trabajadores, los pueblos originarios, entre varios colectivos, en desmedro de otros que se vinculan más directamente con el ámbito cultural, político o militar, por ejemplo?

En cuanto a la presentación efectuada por los editores de Santillana, para el libro de Historia, Geografía y Ciencias Sociales de $1^{\circ}$ Medio, la misma ofrece a sus lectores una invitación a reflexionar sobre el mundo en que vivimos. Se trata de mirar hacia atrás, pero con los ojos del siglo XXI (Gárate, Rodríguez, Castillo y Morales, 2014, p. 3). La Historia es, en definitiva, una "agenda contemporánea", donde el objetivo de su enseñanza se identifica con un fin utilitario vinculado a la comprensión de la propia realidad, al igual que sucede con lo que plantea la reforma española, de la cual se hacen eco varias reformas en Latinoamérica, cuya ley fundamental lo expresa del siguiente modo: "La enseñanza de la Historia tiene como finalidad fundamental que los estudiantes adquieran los conocimientos y actitudes necesarios para comprender la realidad del mundo en que viven, las experiencias colectivas pasadas y presentes, así como el espacio en que se desarrolla la vida en sociedad" (Loe, 2007, citado por Carretero y Montanero, 2008, p. 134).
Carretero y Montanero lo expresan claramente, explicitando las nuevas bases sobre las que se estructura la enseñanza de la Historia:

Más allá de la transmisión de la memoria colectiva y del patrimonio cultural, tiempo, espacio y sociedad deben articularse en las representaciones mentales de los estudiantes para comprender el presente en el que viven, para interpretarlo críticamente. A lo largo de las últimas décadas el papel moralizante y aleccionador en la enseñanza de la Historia ha dejado paso al reconocimiento de su importante función en la formación de ciudadanos críticos y autónomos (Carretero y Voss, 2004). Desde esta nueva concepción, la enseñanza de la Historia no se vertebra ya en torno a los personajes, las fechas y los eventos significativos del pasado. Se pretende que los estudiantes comprendan los procesos de cambio en el tiempo histórico y su influencia en el momento presente, es decir, que aprendan a pensar históricamente. (2008, pp. 134-135)

Lo cual conlleva, según los autores, el desarrollo de habilidades específicas, de allí el énfasis otorgado a los contenidos procedimentales.

En definitiva, la nueva enseñanza de la Historia comporta la desnaturalización de la disciplina. Gestada desde sus inicios para registrar los personajes, las fechas y eventos significativos del pasado, y para que los mismos queden en la memoria colectiva, debe renunciar a la finalidad que le dio vida, para transformarse en una mera herramienta de construcción de ciudadanos activos y críticos. Dos nuevas preguntas surgen ante esta reflexión final: ¿Cuál es la concepción de hombre y de ciudadano que poseen los propulsores de esta "nueva enseñanza"? y ¿por qué debemos privar a las nuevas generaciones del aprendizaje de la Historia en el sentido clásico del término, siendo que la misma pretendía también formar al ciudadano de un orden republicano, legándole la herencia del patrimonio cultural recibido de sus propios antepasados? (Fernández, García 
y Galindo, 2017) ¿Qué diferencia debiera existir entre el ciudadano de los Estados republicanos y este nuevo ciudadano del mundo que se pretende formar, "activo, crítico y autónomo"?5.

Consideramos importante aclarar que en el origen de esta problemática se encuentran los postulados difundidos por organismos internacionales como la Organización de las Naciones Unidas para la Educación, la Ciencia y la Cultura (United Nations Educational, Scientific and Cultural Organization Unesco), que luego de la Segunda Guerra Mundial vienen difundiéndose entre los Estados miembros, con una influencia cada vez mayor, no solo en aspectos prácticos acerca de "cómo educar en un contexto de pobreza", sino teóricos, que se vinculan por ejemplo con el rechazo de los sentimientos de nacionalidad, en pos de un internacionalismo y la creación de un orden mundial, o global, como diríamos en la actualidad, para el cual debe forjarse al nuevo ciudadano del mundo (Unesco, 1948-1949)6. Según el informe de la Comisión Internacional sobre el Desarrollo de la Educación?, dirigida por Edgard Faure y titulado Apprende à être:

Cada uno debería ser conducido a no erigir sistemáticamente sus creencias, sus convicciones, su visión del mundo, sus costumbres y usos en modelos o reglas válidas para todos los tiempos, todos los tipos de civilización y todos los modos de existencia". Lo ideal es "la investigación de verdades nuevas, la transformación de los presupuestos fundamentales de la suerte de los hombres. (Faure, 1972, citado en Caponnetto, 1981, p. 114)

Todos estos cuestionamientos y sus respuestas se hallan presentes de algún modo en los textos analizados (Fernández y Giadrosić, 2015; Landa

$5 \quad$ Cfr. Al respecto, en el Seminario Internacional de Textos escolares de Historia y Ciencias Sociales, la ponencia de Pablo Toro Blanco (2009), donde presenta una visión ilustrativa de la discusión acerca de la concepción de ciudadanía en Chile.

6 En cuanto a la influencia de la Unesco en las políticas educativas nacionales, cfr. Represas (2015, pp. 101-115).

7 Respecto a otros organismos sobre educación y políticas educativas, véase Valle (2012) y Pinto, 2015a/b; Gárate et al., 2014; Mendizábal y Riffo, 2013; Quintana et al., 2012; Latorre, Henríquez y Rocha, 2013), tal como podremos comprobar en la revisión crítica que presentamos a continuación, la cual se abocará, en una primera etapa ${ }^{8}$, específicamente al contenido, luego de haber revisado previamente los fundamentos teóricos sobre los que se sustenta su tratamiento al transformarse en disciplina escolar.

\section{Revisión crítica de los textos (2012-2015)}

\section{El contenido}

La concepción de la Historia y de su enseñanza escolar, propia de los textos del periodo seleccionado, eco a su vez de las Bases Curriculares establecidas por el Estado, tiene una repercusión lógica en los contenidos de cada texto analizado. Dicha repercusión puede percibirse en ciertos indicadores que deben considerarse para evaluar la calidad de estos. En este sentido, desde la perspectiva de su contenido, consideraremos específicamente aquellos que pueden observarse a partir de dos aspectos esenciales, identificados con la forma y el fondo que presentan los diferentes textos. A través del fondo es posible detectar más fehacientemente aquella concepción. Se detallan a continuación los indicadores, que se engloban en cada uno de los aspectos:

1. Fondo: objetividad; equidad en el tratamiento de los temas; información veraz y actualizada; fundamentación de los hechos o fenómenos presentados, de acuerdo con sus causas y efectos; inserción de cada tema en el contexto temporal y espacial correspondiente.

2. Forma: redacción, ortografía, puntuación, sintaxis, transcripción correcta de fuentes, correcto mecanografiado.

8 Cabe acotar que se prevé la continuación de este estudio en una etapa posterior, para atender a las demás variables analizadas en el estudio anterior: estructura y organización del texto, diseño gráfico y metodología. 
OBJeTIVIDAD: Con respecto al fondo, primer factor, destaca por su importancia a la hora de presentar cada uno de los temas. No obstante, es un problema recurrente la visión sesgada en el tratamiento de ciertos temas considerados polémicos, sobre los cuales no existe un consenso universal. Entre ellos podemos destacar:

- Evolucionismo y origen del hombre.

- $\quad$ Situación de la mujer y teoría de género.

- Pacificación de la Araucanía, conflicto mapuche y multiculturalismo.

- Dictadura militar, democracia y derechos humanos.

- Conquista española de América.

Mencionaremos ejemplos de los tres primeros únicamente, para no fatigar al lector, aunque pueden tomarse varios referidos a los temas restantes (Mendizábal y Riffo, 2013, pp.10-82; Latorre et al., 2013, p. 19). En el libro de $7^{\circ}$ Básico de $S M$, se considera el origen del hombre desde una única perspectiva. Se plantea la evolución biológica del homínido, como un hecho probado científicamente, fenómeno que, si bien implica un consenso en la actualidad, ha generado desde el comienzo de su postulación un amplio debate, y se ha aceptado en el ámbito académico como teoría, pero no como algo probado científicamente (Behe, 1999). Nótese, por ejemplo, el relato editorial siguiente, avalado luego por los documentos presentados en el mismo texto, más adelante:

Evidencia cientifica proveniente del estudio de restos fósiles y la investigación genética ha demostrado que grandes primates que hoy conocemos (como el chimpancé, el gorila o el orangután) comparten un ancestro común con el ser humano. El momento exacto en que el árbol evolutivo separó de forma definitiva estas dos ramas no está claro, aunque algunos científicos creen que este ancestro común podría tratarse de un simio africano llamado procónsul (Doc. 1), que habitó los bosques de África oriental hace más de 20 millones de años. Con la aparición de los primeros homínidos comenzó un largo proceso de evolución (Doc. 5) que llevaría eventualmente a la aparición del ser humano moderno, el Homo sapiens. (Landa y Pinto, 2015a, p. 24; énfasis agregado)

Como podemos comprobar, se realiza una afirmación científica de aquello que se supone una certeza, pero que aún suscita mucha discusión entre los científicos de la actualidad, por falta de pruebas fehacientes; además, no existe ninguna referencia a una postura diferente, como por ejemplo el creacionismo científico, y principalmente luego de la aparición, en la década de 1980, de la teoría del Diseño Inteligente, avalada por muchos investigadores críticos de la teoría clásica de Darwin (Ayala, 2007).

Otro caso de sesgo ideológico se relaciona con la situación de la mujer. En el texto de $6^{\circ}$ Básico, se presentan de manera positiva documentos que muestran los avances en cuanto a los derechos de las mujeres, desde su rol tradicional de esposa y madre hasta el rol actual, como profesional, política, empresaria, etc., equiparable al de los varones. La foto de una familia tradicional de principios del siglo XX sirve para graficar los roles convencionales de la mujer que aparecen como obsoletos, en tanto que nuevamente los documentos presentan como válida una sola postura, favorable a los derechos de las mujeres y a la igualdad de género (Fernández y Giadrosić, 2015, pp. 30-31), lo cual excluye la posibilidad de pensamiento crítico y la inferencia de cuál es la mejor situación para la mujer, a partir del análisis de las diferentes perspectivas. Más adelante, el mismo texto vuelve sobre el tema en ocasión de un debate de ideas acerca de la participación política de la mujer. Para ello se presenta en el Doc. 5 un fragmento de un artículo de Internet que se titula "El debate sobre la discriminación positiva de la mujer" y en el cual se afirma:

Suele provocar intensos debates la conveniencia o no de cuotas en torno al $40 \%$ de mujeres $y$ 
sin embargo existe una ausencia absoluta de debate respecto a la cuota entre el 75 y el 100\% de hombres que suele haber en todas las organizaciones: partidos, Iglesia, Ejército, Consejos de Universidad, de Ministros, Municipales, de Administración, Sindicatos, Medios de Comunicación... y que decir de la escasa presencia de mujeres en cualquier ámbito... en torno al 20\% o menos en la literatura, la investigación científica, el profesorado universitario, en el empresariado, el periodismo, en la dirección financiera. Los hombres hacen número en cantidad y ocupan espacios de poder, en calidad. (Boix, 2005, citado por Fernández y Giadrosić, 2015, p. 235; énfasis agregado)

A pesar que es bien marcada la posición ideológica de tendencia feminista, no aparece ninguna otra página de referencia o documento en el texto que sustente una posición alternativa a dicha postura9. Además, al revisar la página web de donde se extrajo el texto (http://www.mujeresenred.net/) se constata que adolece de varios defectos de forma, tales como errores ortográficos, falta de acentos, entre otros, los cuales van en desmedro de su calidad como fuente de información.

En el caso de la demanda mapuche, tratada en el texto de $3^{\circ}$ Medio, se habla de una profundización de sus mecanismos de presión justificando veladamente la quema de fundos y destrucción de maquinaria de propiedad de los empresarios madereros, entendidas como acciones "reivindicativas" y no como verdaderos delitos. Más adelante, se comenta que, como consecuencia de la judicialización del problema a partir de la aplicación de la Ley Antiterrorista, han fallecido varias personas, entre los que se cuentan los "jóvenes comuneros como Alex Lemún, Matías Catrileo, y Jaime Facundo Mendoza

$9 \quad$ Al respecto, se pueden citar dos páginas web con posturas diferentes acerca del feminismo: http://womenagainstfeminism.com/; http://www.ladiesagainstfeminism.com/
Collío, y un carabinero" (Quintana et al., 2012, p. 272). Como podemos observar, los primeros se mencionan con nombre y apellido, en tanto que el último, representante de las fuerzas del orden, permanece en el anonimato, estrategia metodológica apropiada para silenciar una realidad que no conviene mostrar (Mejía, 2009). Se resalta, además, en el mismo texto escolar que se ha producido en forma paralela a estas "reivindicaciones" "una revaloración cultural de los pueblos originarios, la cual se ha traducido en una actualización de rituales y tradiciones, y una reivindicación de sus nombres y apellidos" (Quintana et al., 2012, p. 272). A diferencia de lo sucedido en el siglo XIX, cuando el Estado chileno buscaba homogeneizar la cultura chilena para lograr la unidad con base en una identidad nacional propia, ahora se destacan las diferencias o, como se le denomina actualmente, "la diversidad cultural", sin preocuparse si tal valor afectará la identidad otrora tan ansiada. Resulta interesante que, en un mundo globalizado como el actual, se intente destacar lo que separa a los grupos étnicos dentro del mismo país, en tanto que se asume el multiculturalismo como un valor surgido precisamente como consecuencia de la citada globalización, gracias a la cual se han diluido las características culturales que otorgan identidad a la propia nación (Enkvist, 2011). Nos parece interesante transcribir el comentario de Joan Oleza (2003) cuando afirma, refiriéndose a la relación entre multiculturalismo y globalización:

Me parece plenamente legitima la pregunta de si la aplicación del multiculturalismo al discurso teórico incide más en un movimiento emancipatorio, de puesta en cuestión de una Modernidad opresora, tal como la concibieron desde Max Weber a Foucault, pasando por Adorno y Horkheimer, o una dócil adaptación al mercado, a sus crecientes necesidades de diversificación del consumo, y a las estrategias de neutralización de los grandes conflictos culturales, disgregados en un universo parcelado de diferencias. (p. 6) 
ISSN 0123-1294 | e-ISSN 2027-5358 | Educ.Educ. Vol. 21. No. 1 | Enero-abril de 2018 | pp. 73-92.

Universidad de La Sabana | Facultad de Educación

Respecto de esta problemática que pone en juego la propia identidad cultural, es necesario aclarar que la misma no es privativa de los textos chilenos ni mucho menos de la actualidad. Varios estudios la han detectado en manuales de diversos países y épocas, entre los que cabe citar, en primer lugar, y a propósito de Chile, el artículo titulado "Fabricación heroica y construcción de la memoria histórica chilena (1844-1875)" de Jean Pierre Dedieu, Lucrecia Enríquez y Gabriel Cid Rodríguez (2015), que resalta la importancia de ciertos textos considerados de lectura obligatoria en las escuelas decimonónicas, como instrumento fundamental en la fabricación de los héroes chilenos. En segundo término, deseamos mencionar, a manera de ilustración, la tesis inédita de Piero Colla (2017), que lleva por título "Une heritage impensable. Conscience historique et technologies del 'identité dans la réforme éducative en Suède (1946-1980)", la cual trata de la reforma escolar sueca en la segunda mitad del siglo XX, utilizando como fuente precisamente los manuales escolares. Según este autor: "El manual constituye un objeto accesible a diferentes lecturas, [...] centradas en la confrontación entre representaciones elaboradas del pasado nacional, en las cuales la educación en la admiración o la repugnancia, en la celebración o el desdén, figura como la palanca fundamental". El estudio examina a la vez "la manera en la cual el dato científico es reelaborado, se transforma en una doxa, [y] participa en la construcción de una falsa conciencia" (2017, p. 54).

Más adelante Colla denuncia cómo a partir de la disciplina histórica se gesta una nueva materia, la "educación social", el establecimiento de un sistema de verificación central respecto de la "objetividad" y de los valores fundamentales insertos en los manuales y, por último, la crisis de la noción de "transmisión cultural", como consecuencia de la institucionalización de la doctrina antiautoritaria de la escuela maternal del Estado.

De manera similar, en los textos chilenos, la misma objetividad queda en tela de juicio, cuando paradójicamente las Bases Curriculares se proponen el desarrollo de habilidades de pensamiento crítico, el cual "les entregará a los estudiantes la capacidad de cuestionar de manera autónoma reduccionismos y prejuicios sobre el pasado, [e] identificar sesgos en los puntos de vista" (MIneduc, 2013, p. 197).

EQUIDAD: en el segundo indicador observamos la equidad en el tratamiento de los temas en el texto de $7^{\circ}$ Básico. La problemática del origen del hombre y su evolución biológica y cultural, recientemente analizada, ocupa treinta páginas, en tanto que el legado de las primeras civilizaciones ocupa solo diez. En estas, los párrafos de contenido son muy breves y escuetos, al igual que el tratamiento de la Antigüedad Clásica, que combina el estudio de Grecia y Roma reduciendo drásticamente el papel de la primera en la historia de la humanidad (Landa y Pinto, 2015a, pp. 20-120).

Respecto del cristianismo, el texto se despacha en solo doce líneas, en las cuales no se consignan los aspectos mínimos fundamentales; por ejemplo, los apóstoles son definidos como "los discípulos de Jesús que predicaron su doctrina a su muerte", pero no se menciona que se declararon testigos de su resurrección, lo cual fue fundamental para que se diera esa predicación, ni su número ni los nombres de los más destacados al menos, aspectos que serían clave para identificarlos (Landa y Pinto, 2015a). Lo mismo ocurre con la caída y división del Imperio Romano de Occidente, registrada en solo ocho líneas y con información muy sucinta, tal como podemos observar en el siguiente párrafo:

A partir del siglo III d.C. el Imperio entra en crisis. Las causas que explican su decadencia son múltiples (Doc. 7) y aún se debaten entre historiadores. En el año 395 d.C., para frenar la crisis, el emperador Teodosio dividió el Imperio en Oriente y Occidente, sin embargo, ello no dio resultado. Los pueblos germanos, que durante siglos habitaron en las fronteras del Imperio, comenzaron a influir en la política romana, tanto a través de 
la diplomacia como de la guerra, y paulatinamente se fueron asentando de forma definitiva en territorios del Imperio (Doc. 5). En el año 476 d.C. el germano Odoacro tomó la ciudad de Roma, depuso al emperador y se proclamó rey de Italia. Este evento ha sido interpretado como el final del Imperio romano de Occidente. (Landa y Pinto, 2015a, p. 113)

Se percibe aquí cómo acontecimientos destacados de la historia occidental, como lo son la aparición del cristianismo, la caída del Imperio Romano de Occidente y la división del Imperio, ocupan dos tercios de una página solamente. Cabe inferir, por tanto, que si bien el texto posee un recurso digital complementario, igualmente, en comparación con el tratamiento dado a otras temáticas insertas en el mismo material impreso, la brevedad de la información escrita demuestra la escasa trascendencia que se otorga a estos hechos, cruciales para la formación de Europa y para la comprensión de la historia del Occidente contemporáneo, más aún cuando se trata de un texto de Historia.

VERACIDAD Y ACTUALIZACIÓN DE LA INFORMACIÓN: un tercer indicador se refiere a la veracidad y actualización de la información, característica que, en general, intentan respetar las diferentes editoriales. Sin embargo, varios errores se deslizan en distintas ediciones respecto de ambos criterios, los cuales disminuyen la calidad de los textos. Un ejemplo claro de ello puede observarse en el libro de $7^{\circ}$ Básico antes mencionado (Landa y Pinto, 2015a) en donde se afirma sobre el cristianismo que "en el año 306, el emperador Constantino declaró la libertad de culto mediante el Edicto de Milán" (p. 112), lo cual no es exacto, pues en el año 306 Constantino fue aclamado emperador por las tropas de su padre Constancio Cloro, en tanto que el Edicto de Milán se promulgó recién en el año 313.

Respecto de la actualidad de la información, puede observarse en el texto de $6^{\circ}$ Básico una tabla referida al porcentaje de analfabetismo en Chile, siendo el último dato correspondiente al año 1970, cuando existen datos de censos más recientes como el de 2002, y 2012, que arrojan datos correspondientes a 4,21\% y 2,24\% de analfabetismo (Fernández y Giadrosić, 2015, p. 236).

FUNDAMENTACIÓN DE LOS HECHOS O FENÓMENOS PRESENTADOS, POR SUS CAUSAS O EFECTOS: en cuanto al indicador referido a la fundamentación de los hechos o fenómenos presentados, por sus causas o efectos, hemos podido identificarlo principalmente en aquellas temáticas que son presentadas de manera superficial, aunque sean importantes, o bien en aquellas que resultan polémicas. En este sentido, nótese cómo en el párrafo siguiente del texto de $3^{\circ}$ Medio se plantea la temática que lleva por título "La vida cultural en un contexto autoritario":

Tras el golpe militar de 1973 muchos artistas fueron perseguidos, exiliados del país e incluso asesinados, como el cantautor y director de teatro Víctor Jara. Transcurridos los primeros años de la dictadura militar, la vida cultural comenzó a rearticularse a través de espacios generalmente clandestinos, debido a las restricciones y censuras impuestas por la oficialidad a las expresiones públicas. No obstante, algunos intelectuales se atrevieron a realizar algunas intervenciones artísticas que, aunque furtivas, abrieron cierto grado de expresión social y cultural. (Quintana et al., 2012, p.23010

Podemos percibir que en la primera parte del párrafo el hecho se presenta de manera periodística, sin explicar el contexto en el que se enmarca, signado por el Estado de sitio, ni las causas de las persecuciones, tampoco si los exilios fueron forzados o voluntarios, y ni cómo se explican los asesinatos; mientras que en la segunda parte del texto se justifica claramente la clandestinidad, a causa de la actitud represiva del gobierno hacia la libertad de expresión. En definitiva, se exponen argumentos

10 Respecto de la política cultural durante la dictadura, cabe sugerir el estudio realizado por Errázuriz (2006), titulado "Política cultural del régimen militar chileno (1973-1976)". 
sólidos de los hechos solo en el caso de aquellos que se desean destacar. Ejemplos como estos desmerecen la calidad del texto, al fundamentar adecuadamente solo algunas de las afirmaciones efectuadas, dejando al descubierto la posición ideológica de sus autores y contribuyendo a la vez a presentar una versión sesgada de la realidad. Actitud que, por otra parte, contradice las propias bases curriculares que invitan al estudiante a interpretar críticamente la realidad, lo cual supone la presentación equilibrada de las diferentes versiones de los hechos (MINEDUc, 2013)".

INSERCIÓN DE CADA TEMA EN EL CONTEXTO TEMPORAL Y ESPACIAL CORRESPONDIENTE: en cuanto al último indicador, es posible afirmar que, en términos generales, las casas editoriales se han preocupado por dicha contextualización, estableciendo relaciones entre los hechos sucedidos en Chile con los de los demás países americanos o con los principales sucesos mundiales. Este elemento se contempla como un avance respecto de los textos de la década anterior, que descuidaban este aspecto, ante la preocupación por responder a las exigencias ministeriales. Cabe acotar, no obstante, que dicho avance responde en parte a los cambios establecidos por las mismas Bases Curriculares que, a partir de 2013, incorporaron esta variante, considerada como un aporte positivo de los textos argentinos en nuestro anterior estudio, y muy adecuada para que el alumno pueda relacionar los distintos acontecimientos en forma sincrónica y en distintos espacios geográficos a la vez (Soaje y Orellana, 2013, p. 49)12.

$11 \quad$ Al respecto, compárese con lo expresado en el siguiente párrafo: "El uso de evidencia para argumentar, la rigurosidad y sistematicidad del análisis, el desarrollo de argumentos coherentes y lógicos, entre otros, son algunos ejemplos de criterios que permiten legitimar las distintas visiones sobre un mismo hecho" (MINEDUC, 2013, p. 197).

12 Cfr. lo expresado en el estudio anterior acerca de este aspecto: cabe aclarar, para comprender el contenido de los textos en Argentina, que el currículum de este país en el área de Historia y Ciencias Sociales se halla integrado de tal modo que los alumnos visualizan la historia de una región o país inmersa en un contexto más amplio de referencia. Esto demuestra una preocupación real y válida por lograr que el alumno integre los contenidos que estudia para que estos sean significativos.
Respecto de la forma, segundo aspecto mencionado, el estudio arrojó que, en términos generales, existe una conciencia clara en las editoriales de su importancia y un esfuerzo por guardar la corrección en las distintas aristas de este aspecto. No obstante, se pueden encontrar variados ejemplos de falencias en cada uno de los indicadores mencionados, aunque puede decirse que están en directa relación con la antigüedad del libro, en el sentido en que, mientras más años tiene la edición, menos errores contiene, en tanto que las más modernas poseen mayores falencias. Para ejemplificar podemos mencionar las siguientes fallas, detectadas en el libro de $6^{\circ}$ Básico de SM en su primera edición (Fernández y Giadrosić, 2015): a) errores de redacción (p. 207); b) faltas de concordancia (p. 173); c) repetición de palabras y de artículos (p. 182); d) uso de verbos poco adecuados en la enunciación de las actividades, que se prestan a confusión: creen en vez de elaboren (p. 250), o bien investiguen en vez de averigüen (p. 198); e) párrafos demasiado largos que dificultan la comprensión lectora (p. 208); f) errores de digitación (p. 180); g) signos de puntuación utilizados equivocadamente (p. 207); h) errores en la transcripción de documentos: en Doc. 2 de la página 212 dice Desde en vez de Dése. Todas estas fallas dificultan a veces la lectura y, en último término, desmerecen la calidad del texto.

En definitiva, tanto los aspectos de fondo como los de forma merecen una revisión por parte de las autoridades, ya que sus falencias disminuyen la calidad de los textos y contradicen incluso los criterios expresados en las Bases Curriculares. Una vez más, conviene recordar en este sentido lo expresado por Carretero y Kriger (2014):

La versión de la historia que se distribuye oficialmente a los niños nos da acceso a la voz de quienes producen y validan como tales los conocimientos destinados a conformar nada menos que las primeras imágenes del mundo (Ferro, 1981), a vehiculizar las narraciones oficiales que justifican el presente, los términos de los contratos sociales y las posiciones sociales. (p. 97) 


\section{Conclusiones}

El análisis efectuado nos permite revisar los resultados, considerando, a modo de conclusión, las fortalezas y debilidades de los textos actuales, a partir de la base que nos ha proporcionado el estudio efectuado acerca del periodo 2000-2010. De este modo pretendemos, de acuerdo con el objetivo planteado al comienzo de este trabajo, evaluar en qué medida se han producido cambios en su concepción, traducidos en una mejora en su calidad que justifique el enorme esfuerzo económico realizado año a año por el Estado chileno para proveer, tanto al alumnado como al cuerpo de profesores de establecimientos municipales y subvencionados, de este importante recurso didáctico.

Respecto de las debilidades que han salido a la luz a través del estudio de la variable contenido, se constató, en primer lugar, falencias respecto de la objetividad, resaltando el sesgo ideológico que presenta el tratamiento de ciertos temas polémicos, en los cuales rara vez se consideran equilibradamente las distintas versiones de los mismos. Se observó, en segundo lugar, la falta de equidad, traducida en la superficialidad con que son estudiados algunos hechos o procesos históricos o, por el contrario, la excesiva profundidad que presentan otros; la carencia de una adecuada fundamentación en ciertas problemáticas en las cuales se da por obvio un consenso general, y por otra parte, la presentación de argumentos falaces destinados a justificar comportamientos humanos reprobables; en menor medida, se han registrado también errores que afectan la veracidad de la información que se ofrece, desmereciendo la pretensión de rigor científico del cual depende en gran parte la calidad de los textos. A ello se suman varias fallas de forma, que desmerecen también dicha calidad, en lo que hace a la presentación física del contenido, especialmente, errores de redacción, faltas de concordancia, oraciones demasiado largas, entre otras. No obstante, cabe destacar como fortaleza que se ha valorado positivamente la inserción de las principales temáticas analizadas, en el contexto temporal y espacial correspondiente, aspecto que se vincula con los cambios efectuados en los últimos años a las Bases Curriculares.

La síntesis de lo tratado en nuestro estudio y el análisis de las debilidades y fortalezas de los textos, por una parte, demuestran la continuidad de la mayoría de las falencias registradas en la investigación anterior, y por la otra, nos inducen a volver sobre los fundamentos teóricos del nuevo concepto de la Historia como disciplina, y de su enseñanza, y rescatar las preguntas efectuadas al comienzo de nuestro trabajo. ¿Por qué el alumno debería estudiar esta asignatura a partir de estos textos y no de otros diferentes? Si uno de los objetivos principales es estimular el pensamiento crítico, ¿por qué no fomentar el uso de diferentes textos a partir de los cuales habría más posibilidad de encontrar distintas visiones del pasado y de la actualidad? ¿Cuáles son los criterios según los cuales se priorizan unos contenidos sobre otros, una visión de la Historia sobre otras?

La respuesta, después del análisis efectuado, nos ha llevado a escudriñar los antecedentes de estas innovaciones en la enseñanza de la Historia, reflejadas a su vez en los textos escolares. Ya existen varios estudios que atestiguan sus comienzos en el mundo iberoamericano (Callai, 2008; Carretero y Montanero, 2008; Martínez, Valls, y Pineda, 2009; Zamorano y Villalón, 2008) e incluso algunos autores han levantado la voz de alarma sobre este particular en otros países europeos (Cajani, 2006; Elmersjö, 2014; Enkvist, 2011; Heinrich, 2008). En esta línea se encuentra la tesis doctoral de Piero Colla (2017) que hemos citado anteriormente, dedicada a estudiar la reforma educativa en Suecia, en la segunda mitad del siglo XX, y en particular las características de la nueva enseñanza de la Historia en dicho país, reflejada en sus manuales. La problemática planteada por este lúcido investigador saca a la luz muchas de las características que hemos notado en nuestros libros actuales: la falta de equidad que se traduce en el acento puesto en la Historia contemporánea, en detrimento de los periodos previos, condenados 
al olvido, especialmente la Historia medieval y moderna; la organización temática de la asignatura Historia encorsetada en novedosas categorías sociológicas, que se condicen con la pérdida de la autonomía de la misma como disciplina, y que rebajan a priori la importancia de otras áreas, como las de la Historia militar, la cronología política, la Historia intelectual y cultural, entre otras; la confrontación de ciertos objetivos primordiales de la enseñanza de la Historia, percibida a partir de aquella reforma, como mera "orientación" que apunta a la formación de la ciudadanía versus la transmisión de una herencia cultural, la asimilación de valores sociales fundamentales versus el intelectualismo, el universalismo versus el particularismo. En este juego, el manual de Historia ocupa un lugar como promotor del cambio y no como lugar de resistencia. En palabras del historiador: "En la batalla que el concepto libra contra la imagen, el sentido práctico contra la resonancia del mito, la tradición narrativa que lo determina lo organiza a priori sobre el segundo frente" (Colla, 2017, p. 390). El filtraje progresivo del corpus de contenidos, su reducción a lo útil y la pérdida de perspectiva constituyen otras tantas muestras de la pérdida de identidad de la disciplina, que refleja "la evolución del ethos oficial: del mito optimista del progreso social a aquel del individuo autónomo construyendo su propia historia" (Colla, 2017, p. 391).

En esta misma línea, pero con una lectura más radical de la transformación sufrida por la educación en España gracias a la réplica de la mencionada reforma sueca, un grupo de investigadores ha expresado su preocupación y la necesidad de volver a los fundamentos que dieron origen a la escuela pública, en los siguientes términos:

La escuela debe preparar a los jóvenes para que sean capaces de entender el mundo que les ha tocado vivir y eso solo puede hacerse a partir de la transmisión de la herencia cultural recibida. Entender el mundo no significa adaptarse a él, sino precisamente tomar distancia para poder discutir lo que hay que cambiar. Esa distancia solo puede tener lugar a partir de la conservación y transmisión de las enseñanzas del pasado, protegiendo esa institución intermedia entre la familia y el mundo que [...] permite que el joven se convierta en un ciudadano instruido y autónomo, capaz de actuar y discutir con otros civilizadamente. (Fernández et al., 2017, p. 384)

En definitiva, el contenido, como rescate de la herencia cultural del pasado, y el libro de texto, como vehículo de transmisión de esa herencia, deberían ser considerados nuevamente parte de la discusión acerca de la enseñanza de la Historia, a fin de no transformar el manual escolar en una mera herramienta que, a pesar de sus pretensiones de objetividad, en la práctica replica la función que tradicionalmente ha tenido de expresar la ideología propia del gobierno de turno (Dedieu et al., 2015). Es en la línea de esta afirmación que hallamos la respuesta a los interrogantes anteriormente planteados.

\section{Referencias}

Aurell, J., Balmaceda, C., Burke, P. y Soza, F. (2013). Comprender el pasado: Una historia de la escritura y el pensamiento histórico. Madrid: Akal.

Ayala, F. J. (2007). Darwin y el Diseño Inteligente: creacionismo, cristianismo y evolución. Madrid: Alianza.

Behe, M. (1999). La caja negra de Darwin: el reto de la bioquímica a la evolución. Barcelona: Andres Bello. 
Cajani, L. (2006). Italian history textbooks on the brink of the twenty-first century. En J. Nicholls, School history testbook across cultures: International debates and perspectives (pp. 27-41). Oxford: Symposium Book.

Callai, J. (2008). El difícil diálogo entre los textos escolares de historia y los avances de la historiografía. En M. D. Educación, Seminario Internacional, Textos escolares de Historia y Ciencias Sociales (pp. 104-111). Santiago de Chile: Ministerio de Educación.

Caponnetto, A. (1981). Pedagogía y educación. La crisis de la contemplación en la Escuela Moderna. Argentina: Cruz y Fierro.

Carretero, M. y Kriger, M. (2004). ¿Forjar patriotas o educar cosmopolitas? El pasado y el presente de la historia escolar en un mundo global. En M. Carretero y J. Voss, Aprender y pensar la Historia (pp. 71-98). Buenos Aires: Amorrortu.

Carretero, M. y Montanero, M. (2008). Enseñanza y aprendizaje de la Historia: aspectos cognitivos y culturales. Cultura y Educación. Fundación Infancia y Aprendizaje, 20(2), 133-132. Recuperado de http://www.ub.edu/ histodidactica/images/documentos/pdf/ensenanza_aprendizaje_historia.pdf

Centro de Microdatos (2013). Servicio de implementación de sistema de seguimiento al uso de texto escolares en uso durantel el 2013. Universidad de Chile, Departamento de Economía, Santiago de Chile.

Claro, S. (2015). Chile lidera ranking educacional en América Latina Avances y desafíos para Chile de acuerdo a TERCE. Punto de Referencia, 396 (marzo). Centro de Estudios Públicos. Recuperado de https://www.cepchile. cl/chile-lidera-ranking-educacional-en-america-latina-avances-y-desafios/cep/2016-03-04/101135.html

Colla, P. S. (2017). Une heritage impensable. Conscience historique et technologies del'identité dans la réforme éducative en Suède (1946-1980). Tesis doctoral, L'École des hautes études en sciences sociales [EHESS], Francia.

Dedieu, J. P., Enríquez, L. y Cid Rodríguez, G. (2015). Fabricación heroica y construcción de la memoria histórica chilena (1844-1875). Caravelle. Cahiers du monde hispanique et luso-brésilien, 104, 47-70.

Dirección de Presupuestos [Dipres]. (2016). Ley de presupuesto año 2016 Ministerio de Educación. Santiago de Chile: Gobierno de Chile. Dirección de presupuestos. Recuperado de http://www.dipres.gob.cl/595/articles-140571_doc_pdf.pdf

Elmersjö, H. (2011). The meaning and use of "Europe" in Swedish history textbooks, 1910-2008. Education inquiry, 2(1), 61-78.

Enkvist, I. (2011). ¿Para quién es positiva la ideología el multiculturalismo? En La buena y la mala educación: Ejemplos internacionales (pp. 148-159). Madrid: Encuentro.

Errázuriz, L. (2006). Política cultural del régimen militar chileno (1973-1976). Aisthesis, 40, 62-78.

Eyzaquirre, B. y Fontaine, F. (1997). El futuro en riesgo: nuestro textos escolares. Santiago de Chile: Centro de Estudios Públicos. 
ISSN 0123-1294 | e-ISSN 2027-5358 | Educ.Educ. Vol. 21. No. 1 | Enero-abril de 2018 | pp. 73-92.

Universidad de La Sabana | Facultad de Educación

Fernández, C.y Giadrosić, G. (2015). Historia, Geografía y Ciencias Sociales 6 Básico. Santiago de Chile: SM.

Fernández, C., García, O. y Galindo, E. (2017). Escuela o barbarie: entre el neoliberalismo salvaje y el delirio de la izquierda. Madrid: Akal.

Gárate, M., Rodríguez, C., Castillo, S. y Morales, J. (2014). Historia, Geografía y Ciencias Sociales $1^{\circ}$ Medio. Santiago de Chile: Santillana del Pacífico.

González, L. (2005). De la múltiple utilización de la Historia. En C. Pereyra et al., Historia ¿para qué? (pp. 53-74). México: Siglo XXI.

Heinrich, K. (2008). ¿Cómo reflejan los textos escolares de historia la investigación histórica? Algunos hallazgos desde Alemania. Seminario Internacional "Textos escolares de Historia y Ciencias Sociales" (pp. 119-127). Santiago de Chile: Ministerio de Educación.

Landa, L. y Pinto, V. (2015a). Historia, Geografia y Ciencias Sociales $7^{\circ}$ Básico. Santiago de Chile: SM.

Landa, L.y Pinto, V. (2015b). Historia, Geografía y Ciencias Sociales $8^{\circ}$ Básico. Santiago de Chile: SM.

Latorre, I., Henríquez, M. y Rocha, P. (2013). Historia, Geografia y Ciencias Sociales $4^{\circ}$ Medio. Santiago de Chile:Zig-Zag.

León, A. y Alvarado, P. (2011). Territorio, familia y héroe: un análisis de textos escolares chilenos. Educación y Educadores, $14(1), 13-25$.

Martínez, N.,Valls, R.y Pineda, F. (2009). El uso del libro de texto de Historia de España en Bachillerato: diez años de estudio, 1993-2003 y dos reformas (LGE-LOGSE). Didáctica de las Ciencias Experimentales y Sociales, 23, 3-35.

Mejía, W. (2009). Análisis de los contenidos de los textos escolares de Historia y Ciencias Sociales. En Seminario Internacional de Textos Escolares (pp. 487-527). Santiago de Chile: Ministerio de Educación.

Mendizábal, M. A. y Riffo, J. (2013). Historia, Geográfia y Ciencias Sociales $2^{\circ}$ Medio. Santiago de Chile: SM.

Ministerio de Educación (2013). Bases Curriculares de $7^{\circ}$ básico a $2^{\circ}$ medio. Historia, Geografía y Ciencias Sociales. Santiago de Chile: Gobierno de Chile. Recuperado de http://www.curriculumenlineamineduc.cl/605/articles-30013_recurso_17_06.pdf

Ministerio de Educación (s.f). Política de textos escolares. Santiago de Chile: Gobierno de Chile. Recuperado de http://www.textosescolares.cl/usuarios/tescolares/File/Folleto_Politica_nuevaversion.pdf

Oleza, J. (2003). Multiculturalismo y globalización: Pensando historicamente el presente desde la literatura. Prosopopeya: Revista de Crítica Contemporánea, 4, 133-156.

Quintana, S., Castillo, S., Pérez, N., Moyano, C. y Thielemann, L. (2012). Historia, Geografía y Ciencias Sociales $3^{\circ}$ Medio. Santiago de Chile: SM.

Represas, N. (2015). Política educativa de la Unesco: un modelo de análisis supranacional. Bordón, 67(2), 101-115. 
Soaje, R. (2012). Estudio de los textos de Historia y de Ciencias Sociales chilenos entre 2000 y 2010. Educación y Educadores, 23-41.

Soaje, R. y Orellana, P. (2013). Textos escolares y calidad educativa. Estudio de la calidad de textos escolares entregados por el Mineduc. Santiago de Chile: Universitaria.

Toro, P. (2009). Concepciones de ciudadanía en Chile a través de textos escolares de Historia y Ciencias Sociales (c. 1880-c. 1930). En Seminario Internacional Textos Escolares de Historia y Ciencias Sociales (pp. 250-262). Santiago de Chile: Ministerio de Educación.

Unesco. (1948-1949). Hacia un entendimiento mundial (vol. VI, secc. II). París: Unesco.

Valle, J. (2012). La política educativa supranacional: un nuevo campo de conocimiento para abordar las políticas educativas en un mundo globalizado. Revista Española de Educación Comparada, 20, 109-144.

Zamorano, A. y Villalón, G. (2008). La Nueva Historia y su aplicación a los textos escolares. En M. d. Educación, Seminario Internacional Textos Escolares de Historia y Ciencias Sociales (pp. 112-118). Santiago de Chile: Ministerio de Educación. 
